\title{
Hexamethyldisiloxane film as the bottom antireflective coating layer for ArF excimer laser lithography
}

\author{
Hsuen-Li Chen and Lon A. Wang
}

\begin{abstract}
We demonstrate a new bottom antireflective coating (BARC) layer for ArF excimer laser lithography. The antireflective layer is composed of hexamethyldisiloxane (HMDSO) film, which is deposited by the conventional electron cyclotron resonance-plasma-enhanced chemical-vapor deposition process. We obtain the appropriate HMDSO films for BARC layers by varying the gas-flow rate ratio of oxygen to HMDSO. Such a process has several advantages: high deposition rate, low process temperature, easy film removal, and reduced cost. Measured reflectances of less than $0.5 \%$ on both $\mathrm{Al}-\mathrm{Si}$ and silicon crystal substrates have been achieved and agree well with the simulated reflectances. The swing effect is shown to be significantly reduced by addition of the HMDSO-based BARC layer. (C) 1999 Optical Society of America

OCIS codes: $\quad 220.3740,310.1210,310.1620,310.6860,310.1860$.
\end{abstract}

\section{Introduction}

In the recently published Semiconductor Industry Association road map, ArF excimer laser-based technology leads optical lithography to the generation of 130-nm feature size. ${ }^{1}$ The problems of critical dimension control caused by highly reflective substrates are far more serious in the 193-nm wavelength regime than in the previous g-line (436$\mathrm{nm})$ and $i$-line (365- $\mathrm{nm})$ regimes. The light reflected from such substrates can result in both swing and notching effects in the coated resist layer, which can cause variation in exposure dosage. The swing effect refers to the sinusoidally varying reflectivity as a function of resist thickness; the notching effect is due to the light reflected from the topography of the substrate that becomes concentrated at certain positions of the resist. ${ }^{2-4}$ It is therefore important to find a high-performance antireflective coating layer that will work in this spectral regime. There are two primary approaches to reducing reflection. One is to use a top antireflective coating (TARC) layer, and the

The authors are with the Institute of Electro-Optical Engineering, National Taiwan University, Taipei, Taiwan. The e-mail address for L. A. Wang is lon@ccms.ntu.edu.tw.

Received 12 January 1999; revised manuscript received 26 April 1999.

0003-6935/99/224885-06\$15.00/0

(C) 1999 Optical Society of America other is to use a bottom antireflective coating (BARC) layer. These coated layers are designed to minimize the reflection from the air-resist (TARC) and the resist-substrate (BARC) interfaces. A BARC layer can be used to reduce both swing and notching effects, whereas a TARC layer can only alleviate the swing effect and thus has limited applicability. ${ }^{5}$ BARC layers have been classified into two parts. Type I consists of organic BARC materials and is widely used in integrated circuits. An organic BARC material that is more absorptive than the overlaid resist is usually spread onto a substrate by direct spinning. Generally it is difficult to find an organic material that can match the various highly reflective substrates that are often encountered in integrated circuits processing. In addition, the capability for step coverage and the accuracy of thickness control by spin coating are poor for Type I materials. ${ }^{6}$ Type II comprises inorganic BARC materials, which are deposited by the conventional chemical-vapor deposition (CVD) method. It has the advantage of composition and thickness tunability, thus providing the possibility of completely eliminating the reflectance from various highly reflective substrates. Materials such as amorphous carbon, silicon carbide, silicon oxynitride, and titanium oxide have reportedly been used in 248-nm lithographic applications, and the silicon oxynitride material has been used at 193 $n m .{ }^{6-11}$ One can optimize the optical constant of an inorganic BARC material by changing the gas-flow 
rate ratio of its constituents, and its thickness can be controlled well during CVD. However, the processing required for an inorganic BARC material is more complicated and sometimes more expensive than for an organic material. Therefore it is desirable to combine the processing and material advantages of both types I and II to form a BARC layer in such a way that composition and thickness control and convenience of processing can be obtained simultaneously.

Here we demonstrate a new BARC layer for ArF excimer laser lithography. The antireflective layer is composed of a hexamethyldisiloxane (HMDSO) film deposited by the conventional electron cyclotron resonance-plasma-enhanced CVD process. Note that HMDSO is a liquid and is relatively cheap compared with the conventional gases that are usually used. Additionally, such a process has several advantages: high deposition rate, low process temperature, easy film removal, and reduced cost.12-14 HMDSO films have been used for electrical insulation, moisture sensing, and corrosion protection. ${ }^{12}$ However, other than in the visible-wavelength range, ${ }^{13}$ the characteristics of HMDSO, especially in the deep-ultraviolet regime, have seldom been reported. Here we vary the gas-flow rate ratio of oxygen-HMDSO to obtain HMDSO films with different compositions and characterize their optical properties at a 193-nm wavelength. We then analyze the composition of the fabricated HMDSO film by utilizing an x-ray photoelectron spectrometer. ${ }^{15}$ Optical characteristics are simulated by use of an admittance diagram, and the simulation results thus obtained are used as the basis for construction of HMDSObased BARC layer for two exemplary $c-\mathrm{Si}$ and $\mathrm{Al}-\mathrm{Si}$ substrates. The swing effects in resist before and after a BARC layer is added are compared. We also discuss the practicability of using HMDSO films as BARC layers for other highly reflective substrates. The etching property of HMDSO film deposited underneath a commercial 193-nm single-layer resist is also characterized.

\section{Optimization of Optical Characteristics of a Bottom Antireflective Coating Layer}

We utilize an admittance diagram ${ }^{16}$ to optimize the optical characteristics of a BARC layer. As shown in Fig. 1, point $\mathrm{S}$ represents a highly reflective substrate with optical constants $n_{s}$, and $k_{s}$, and point $\mathrm{M}$ is the medium above the BARC layer with optical constants $n_{m}$, and $k_{m}$, which in general is a resist. For normal incidence, the Fresnel reflection coefficient can be expressed as

$$
\rho=\frac{N_{m}-N_{s}}{N_{m}+N_{s}}=\frac{\left(n_{m}-i k_{m}\right)-\left(n_{s}-i k_{s}\right)}{\left(n_{m}-i k_{m}\right)+\left(n_{s}-i k_{s}\right)} .
$$

If we can make the effective $N_{s}$ approach $N_{m}$ by adding a BARC layer, the reflection between medium and substrate will be reduced. As shown in Fig. 1, traces $\mathrm{A}$ and $\mathrm{B}$ represent different BARC materials that result in $\rho \cong 0$. Because a BARC layer will be

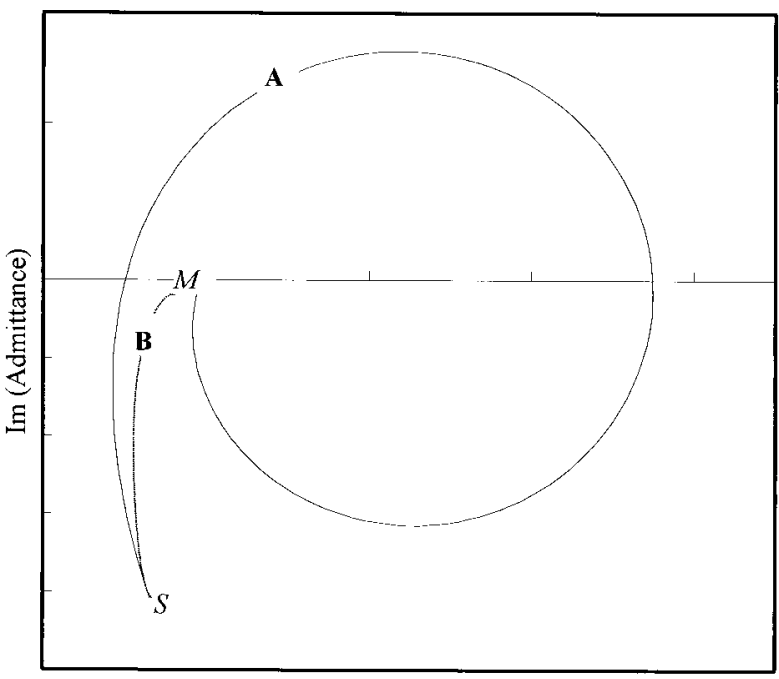

$\operatorname{Re}$ (Admittance)

Fig. 1. Admittance loci for different BARC layers.

etched away after the resist is developed, the layer should be as thin as possible to facilitate film removal. Therefore, although both BARC materials A and $\mathrm{B}$ can be used to reduce reflectance, material $\mathrm{B}$ is better because it is thinner. Let us consider a numerical example. The reported optical constants of the resist (TER-1, Microlithography Chemical Corporation) and the silicon crystal substrate are $N_{R}$ $(1.490,0.018)$ and $N_{\mathrm{Si}}(0.90,2.65)$, respectively. ${ }^{11}$ Assume that the optical constants of materials $\mathrm{A}$ and B are $N_{A}(1.668,0.233)$ and $N_{B}(1.716,0.517)$, respectively. Then the reflectance between resist and substrate can be reduced to less than $1 \%$ when the thicknesses are 86.7 and $27.9 \mathrm{~nm}$ for materials $\mathrm{A}$ and $\mathrm{B}$, respectively, an apparent advantage for material B.

\section{Experimental Setup}

We deposited the HMDSO films by electron cyclotron resonance-plasma-enhanced CVD. The dc bias was fixed at $800 \mathrm{~V}$ during the deposition, and the substrate was not heated. The parameters used for the deposition of HMDSO films are summarized in Table 1. The transmittance and reflectance spectra were measured with an optical spectrometer (Hitachi U3501). The optical constant of a HMDSO film was obtained by the R-T method, ${ }^{17}$ and the composition of the film was analyzed with an x-ray photoelectron spectrometer (Perkin-Elmer, PHI 1600). To study the etching characteristics of HMDSO, we etched var-

Table 1. Parameters Used for Deposition of HMDSO Films

Chamber pressure

Supplied gases

Microwave power

dc bias of rf power supply

Pressure during deposition

Substrate temperature
$5 \times 10^{-6}$ Torr

HMDSO, $\mathrm{O}_{2}$, and Ar

$720 \mathrm{~W}$

$800 \mathrm{~V}$

$7 \times 10^{-3}$ Torr

Room temperature 


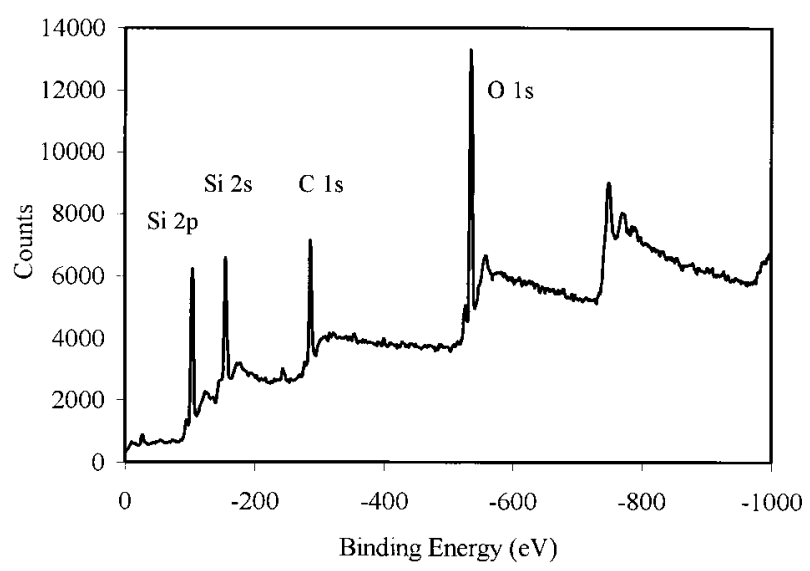

Fig. 2. X-ray photoelectron spectrum of a HMDSO film.

ious substrates that contained HMDSO films as BARC layers, using $\mathrm{CHF}_{3}$ as a reactive agent.

\section{Results and Discussion}

A. X-Ray Photoelectron Spectroscopy of Hexamethyldisiloxane Films

The chemical formula for the HMDSO monomer is $\left(\mathrm{Me}_{3} \mathrm{Si}\right)_{2} \mathrm{O}$, where $\mathrm{Me}$ is composed of $\mathrm{C}$ and $\mathrm{H}$ atoms $\left(\mathrm{Me}=\mathrm{CH}_{3}\right)$. Figure 2 shows a wide-scan x-ray photoelectron spectrometer spectrum of a HMDSO film. Within the detection limit of the x-ray photoelectron spectrometer, only the elements $\mathrm{C}, \mathrm{Si}$, and $\mathrm{O}$ in the HMDSO films were observed. The $\mathrm{C} 1 s, \mathrm{O} 1 s$, and $\mathrm{Si}$ $2 p$ peaks were chosen as indications for the composition ratio of a HMDSO film. ${ }^{15}$ The atom concentrations of the films deposited at different $\mathrm{O}_{2} / \mathrm{HMDSO}$ gas-flow rate ratios are shown in Fig. 3. The $\mathrm{C}$ concentration decreased and the $\mathrm{O}$ increased first rapidly and then slowly, and the Si decreased slowly, as the gas-flow rate ratio increased. When the $\mathrm{O}_{2} /$ HMDSO gas flow rate ratio decreased to 3.75 , the $\mathrm{C}$ concentration decreased to $2.05 \%$, and the concentrations ratio of $\mathrm{O} / \mathrm{Si}$ reached 1.86. We then could obtain a silica $\left(\mathrm{SiO}_{2}\right)$-like HMDSO film that had low

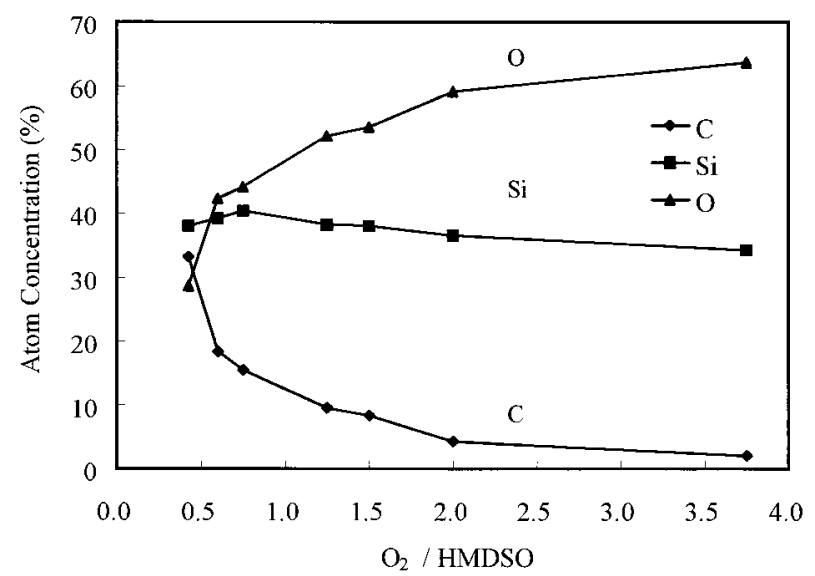

Fig. 3. Composition dependence of HMDSO films on gas-flow rate ratio.

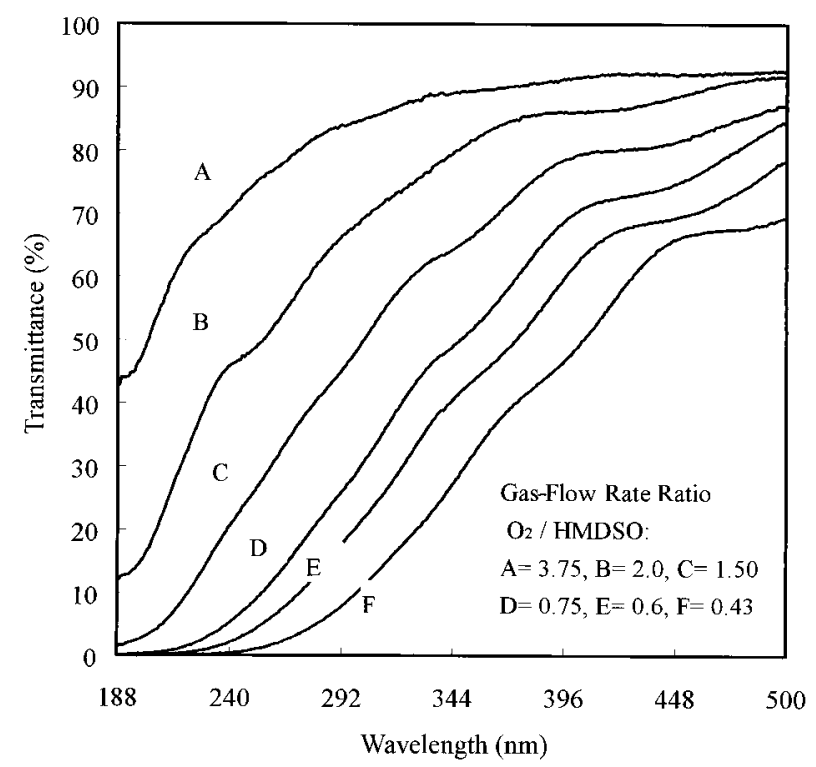

Fig. 4. Transmission dependence of HMDSO films on gas-flow rate ratio.

absorption in the deep-ultraviolet regime. It is therefore convenient to obtain various compositions of a HMDSO film by varying the $\mathrm{O}_{2} / \mathrm{HMDSO}$ flowrate ratio.

\section{B. Optical Properties of Hexamethyldisiloxane Films}

As shown in Fig. 4, we measured the transmittance from the visible to the deep UV spectral regimes of HMDSO films deposited at various $\mathrm{O}_{2} / \mathrm{HMDSO}$ gasflow rate ratios. The higher the ratio, the lower the carbon concentration, which leads to a higher $\mathrm{O} / \mathrm{Si}$ ratio. ${ }^{15}$ This results in less absorption, and therefore the transmittance increases with the ratio, especially in the deep-ultraviolet regime. The optical constants of various HMDSO films measured at 193 $\mathrm{nm}$ are shown in Fig. 5. When the gas-flow rate ratios increased, the extinction coefficient $(k)$ first decreased rapidly and then became saturated. Conversely, the refractive index $(n)$ decreased slowly with

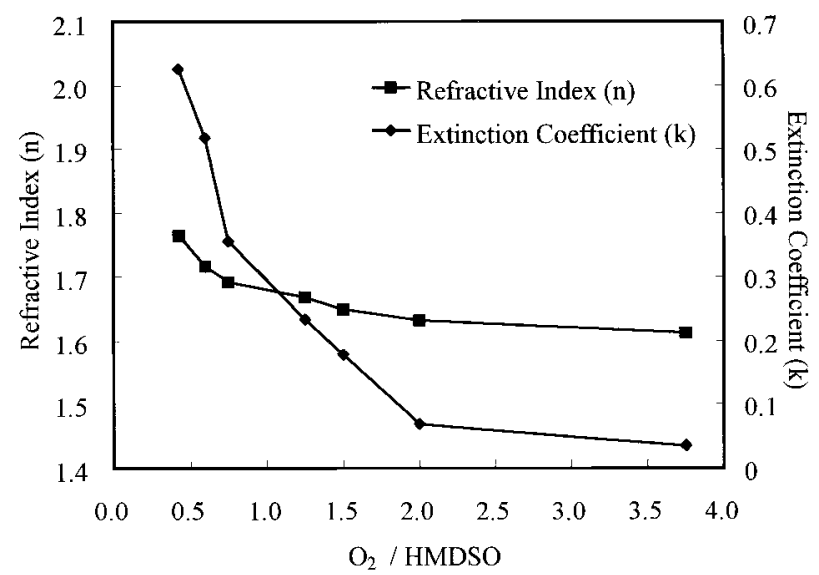

Fig. 5. Optical constant dependence of HMDSO films on gas-flow rate ratio. 


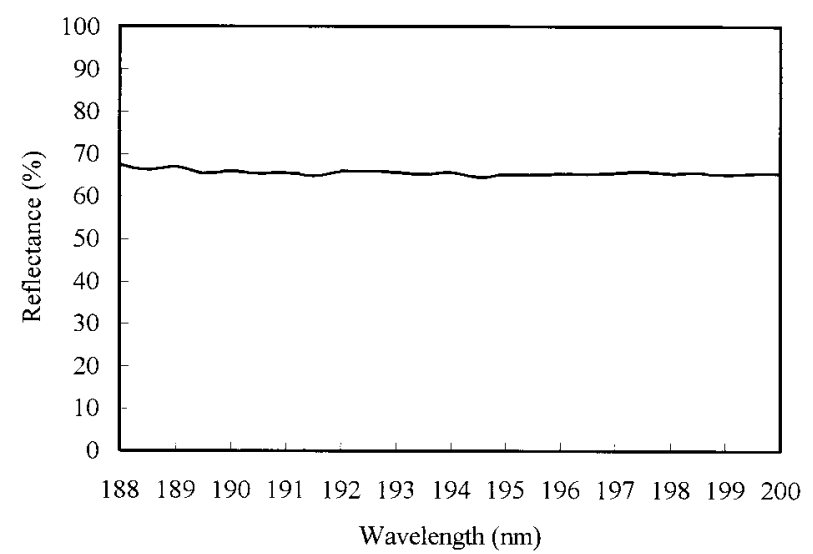

(a)

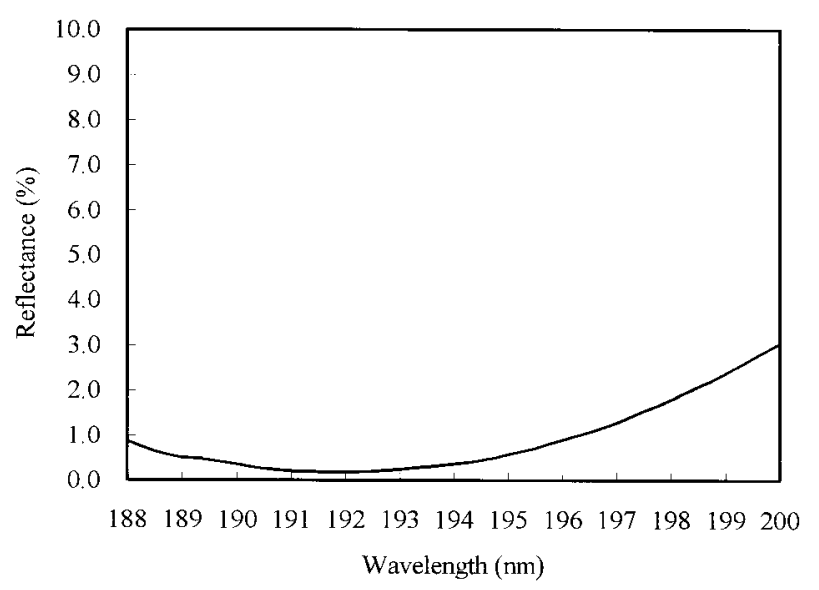

(b)

Fig. 6. Reflection spectrum of a Si substrate (a) before and (b) after addition of a BARC layer.

the ratio. To meet the requirement of a desirable BARC material, refractive index $n$ and extinction coefficient $k$ of such a film should be well controlled. By changing the flow rate ratio of gases $\mathrm{O}_{2}$ to HMDSO, we have been able to obtain a HMDSO film with suitable optical constants.

\section{Hexamethyl/disiloxane Film as a Bottom Antireflective Coating Layer}

\section{Antireflective Coating Layer for a Silicon Crystal Substrate}

To reduce the reflectance from a Si crystal substrate, one should have the combined effective optical constant of the Si crystal substrate and the BARC layer as close as possible to the optical constant of the incident medium $\left[N_{\text {air }}=(1,0)\right]$. According to this rule, a HMDSO film is fabricated to have the same measured optical constant $(1.716,0.517)$ as the BARC layer. An admittance diagram shows that the optimal thickness should be $22.2 \mathrm{~nm}$. As shown in Fig. 6(a), the reflectance of a Si crystal substrate was $\sim 66 \%$ at $193 \mathrm{~nm}$. After a BARC layer with a thickness of $22.2 \mathrm{~nm}$ was added, the reflectance decreased to be less than $0.23 \%$ at $193 \mathrm{~nm}$, as shown in

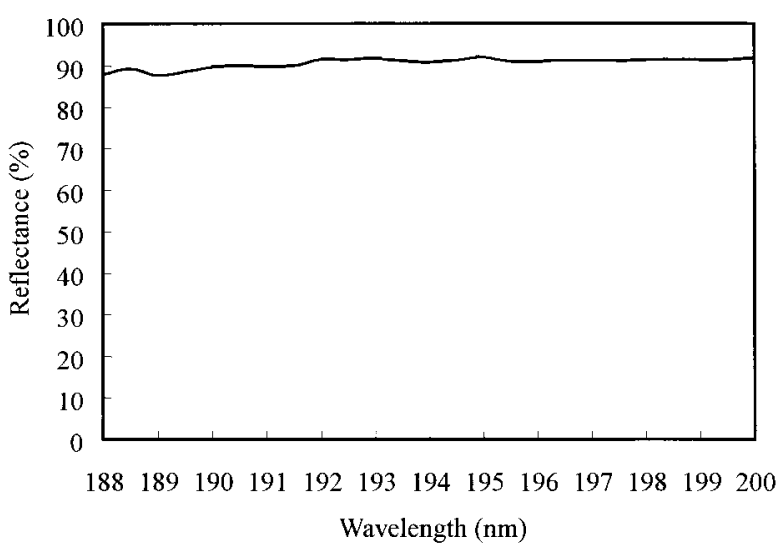

(a)

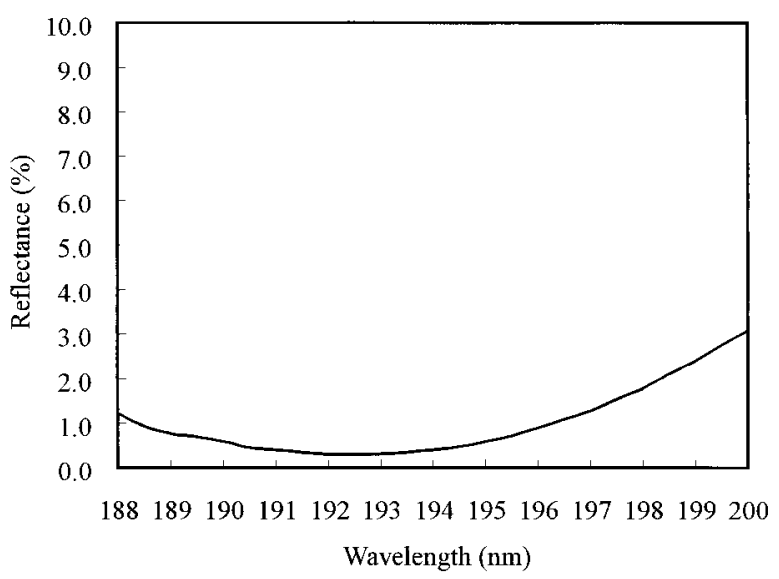

(b)

Fig. 7. Reflection from $\mathrm{Al}$ film coated upon a Si substrate (a) without and (b) with a BARC layer.

Fig. 6(b). The measured result is consistent with the simulated result.

\section{Antireflective Coating Layer for an Al Film Deposited upon Si Crystal Substrate}

As shown in Fig. 7(a), the reflectance of a 300-nmthick $\mathrm{Al}$ film coated upon a $\mathrm{Si}$ substrate was measured to be $\sim 92 \%$ at $193 \mathrm{~nm}$. The HMDSO film with the optical constants $(1.716,0.517)$ was again chosen as the BARC layer. The optimized thickness of the BARC layer was $\sim 20.6 \mathrm{~nm}$. After the BARC layer was added, the reflectance was measured as $0.30 \%$ at $193 \mathrm{~nm}$, as shown in Fig. 7(b), which is also in good agreement with the simulated result.

\section{Reduction of Swing Effects in Resist}

The swing effect caused by optical interference between the fields reflected from both the air-resist and the resist-substrate interfaces introduces the issue of control of critical dimensions in optical lithography. ${ }^{18}$ The reflectance swing curves of TER-1 resist coated onto a Si wafer are simulated and measured as shown in Fig. 8(a). The reflectance exhibits a sinusoidal variation from $\sim 5 \%$ to $\sim 40 \%$ for resist thicknesses ranging from 300 to $600 \mathrm{~nm}$. To reduce the reflec- 


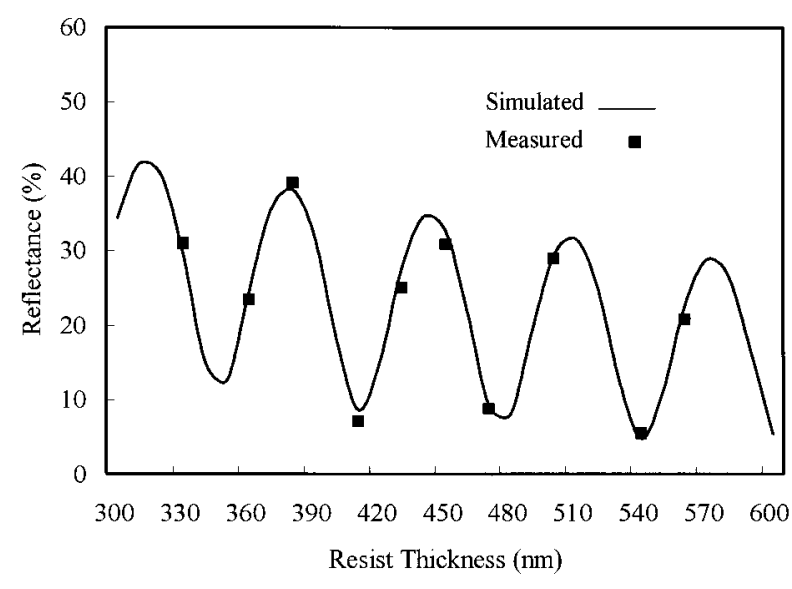

(a)

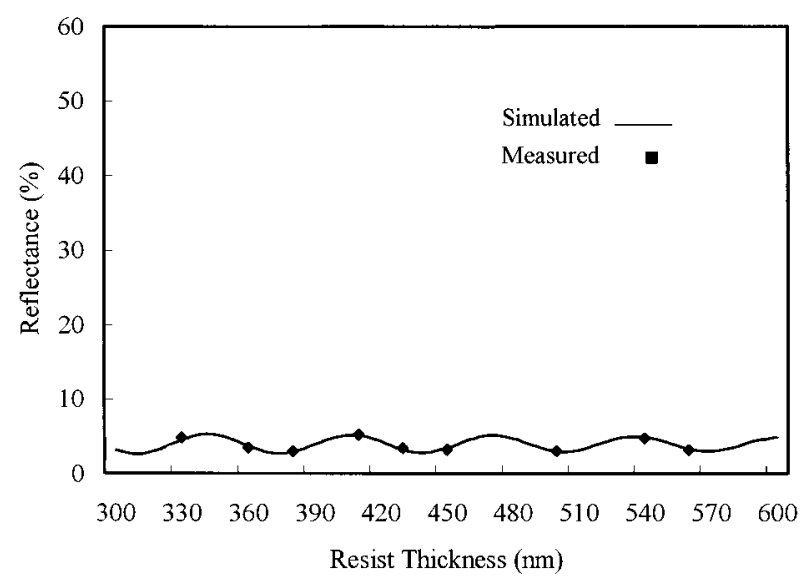

(b)

Fig. 8. Reflectance swing curves of the TER-1 resist coated upon a Si wafer (a) without and (b) with a BARC layer.

tance at the resist-Si substrate interface we chose a HMDSO film with optical constants $(1.716,0.517)$ as the BARC layer. The optimized thickness of the BARC layer was $\sim 28.6 \mathrm{~nm}$. After the BARC layer with the same thickness was added, the reflectance from the resist-BARC-silicon substrate could be decreased to $0.22 \%$. In addition, the reflectance swing curve was simulated and measured as shown in Fig. 8 (b), which has a sinusoidal variation from $\sim 2.5 \%$ to $\sim 5 \%$ for resist thicknesses from 300 to $600 \mathrm{~nm}$. The results show that the HMDSO-based BARC layer can significantly reduce the swing effect in the resist. We expect that the capability for controlling dimensions in optical lithography can be improved by use of such a BARC layer.

\section{Antireflective Coating Layer for Other Highly Reflective Substrates}

We evaluated the practicability of using HMDSO films as BARC materials for other highly reflective substrates such as polysilicon, tungsten silicide, and aluminum silicon by simulating their optical performance, and the results are summarized in Table 2. The optical constants of these highly reflective mate-
Table 2. Characteristics of HMDSO-Based BARC Layers for Highly Reflective Substrates

\begin{tabular}{|c|c|c|c|}
\hline Substrate & Poly-Si ${ }^{a}$ & $\mathrm{~W}-\mathrm{Si}^{b}$ & $\mathrm{Al}-\mathrm{Si}^{c}$ \\
\hline $\begin{array}{l}\text { Optical constants at } 193 \mathrm{~nm} \\
\text { (Ref. 11) }\end{array}$ & \multicolumn{3}{|c|}{$(0.90,2.30)(1.15,2.24)(0.15,1.15)$} \\
\hline $\begin{array}{l}\text { Reflectance from resist- } \\
\text { substrate }(\%)\end{array}$ & 51.2 & 42.8 & 82.4 \\
\hline $\begin{array}{l}\text { Reflectance from resist-BARC } \\
\text { layer substrates after a } \\
\text { BARC layer is added }(\%)\end{array}$ & 0.11 & 0.01 & 0.45 \\
\hline Thickness of BARC layer (nm) & 27.0 & 27.0 & 21.1 \\
\hline
\end{tabular}

rials at $193 \mathrm{~nm}$ were obtained from Ref. 11. Such results shown in Table 2 indicate that HMDSO film will reduce the reflectance from the interface of the resist-highly reflective materials to less than $0.5 \%$ with a thickness of less than $30 \mathrm{~nm}$. The HMDSO films are therefore expected to have great potential for use as BARC materials for these highly reflective materials.

\section{Etching Properties of Hexamethyldisiloxane Films}

The BARC layer needs to be etched away after resist development. During the etching process the resist pattern over the BARC layer should remain as nearly intact as possible to avoid variation in the critical dimensions. Because the major constituents of HMDSO films are $\mathrm{Si}, \mathrm{O}$, and $\mathrm{C}$, as mentioned above, $\mathrm{CHF}_{3}$ gas was chosen as the reactive agent in the electron cyclotron resonance-reactive-ion-etching process. The measured etching rates of the HMDSO film and the resist (TER-1) were 31 and $20 \mathrm{~nm} / \mathrm{min}$, respectively. As described in Subsection 4.C.3, a HMDSO film with thickness of $28.6 \mathrm{~nm}$ will reduce the reflectance from the resist-silicon substrate interface to less than $0.22 \%$. If we remove the HMDSO film, the thickness of resist over the HMDSO film will be reduced by $\sim 18.5 \mathrm{~nm}$. In general, because the initial thickness of resist can be more than $500 \mathrm{~nm}$, such a decrease in thickness is acceptable.

\section{Conclusions}

A HMDSO film deposited by the conventional electron-cyclotron resonance-plasma-enhanced CVD process has been found to be an appropriate new BARC material for ArF excimer laser lithography. One can control the composition and optical characteristics of HMDSO films by varying the $\mathrm{O}_{2} / \mathrm{HMDSO}$ gas-flow rate ratio. The fabrication process can be operated at room temperature, and the deposition rate is high. After resist development, the HMDSO film can easily be removed by the conventional reactive-ion-etching process. The measured reflectance on both $\mathrm{Al}-\mathrm{Si}$ and Si crystal substrates is less than $0.5 \%$ and is consistent with the simulation result obtained by the admittance diagram method. 
The swing effect in the single-layer resist coated onto a Si substrate is also significantly reduced by addition of the HMDSO-based BARC layer. A HMDSO film also has a great potential to be used as BARC layer upon other high-reflectance substrates such as polysilicon, tungsten silicide, and aluminum silicon.

\section{References}

1. P. Gargini, J. Glaze, and O. Williams, "The SIA's 1997 national technology roadmap for semiconductors," Solid State Technol. 41, 73-76 (1998)

2. J. R. Sheats and B. W. Smith, Microlithography Science and Technology, (Marcel Dekker, New York, 1998).

3. C. M. Dai and S. H. Liu, "Rough polysilicon film as a highperformance antireflective layer for sub-half-micron photolithography,” Jpn. J. Appl. Phys. 34, 6611-6614 (1995).

4. C. A. Mack, "Antireflective coatings," Microlithogr. World 6(3), 29-30 (1997).

5. J. Sturtevant and B. Roman, "Antireflection strategies for advanced photolithography," Microlithogr. World 4(4), 13-21 (1995).

6. Y. Tani, H. Mito, Y. Okuda, Y. Todokoro, T. Tatsuta, M. Sawai, and O. Tsuji, "Optimization of amorphous carbon-deposition antireflective layer for advanced lithography," Jpn. J. Appl. Phys. 32, 5909-5913 (1993).

7. T. Ogawa, M. Kimura, Y. Tomo, and T. Tsumori, "Novel ARC optimization methodology for $\mathrm{KrF}$ excimer laser lithography at low $\mathrm{K}_{1}$ factor," in Optical/Laser Microlithography V, J. D. Cuthbert, ed., Proc. SPIE 1674, 362-375 (1992).

8. T. Gocho, T. Ogawa, M. Muroyama, and J. Sato, "Chemical vapor deposition of anti-reflective layer film for excimer laser lithography,” Jpn. J. Appl. Phys. 33, 486-490 (1994).

9. T. Ogawa, T. Gocho, H. Nakano, and M. Tsukamoto, "Hydrogenated silicon-oxynitride film antireflective layer for optical lithography," Jpn. J. Appl. Phys. 36, 3775-3785 (1997).

10. B. H. Jun, S. S. Han, K. S. Kim, J. S. Lee, Z. T. Jiang, B. S. Bae, K. No, D. W. Kim, H. Y. Kang, and Y. B. Koh, "Titanium oxide film for the bottom antireflective layer in deep ultraviolet lithography," Appl. Opt. 36, 1482-1486 (1997).

11. T. Ogawa, A. Sekiguchi, and N. Yoshizawa, "Advantages of a SiOxNy:H antireflective layer for ArF excimer laser lithography,” Jpn. J. Appl. Phys. 35, 6360-6365 (1996).

12. D. Korzec, D. Theirich, F. Werner, K. Traub, and J. Engemann, "Remote and direct microwave plasma deposition of HMDSO films," Surf. Coating Technol. 74, 67-74 (1995).

13. R. Rochotzki, M. Arzt, F. Blaschta, E. Krebig, and U. H. Poll, "Optical properties of plasma polymer films," Thin Solid Films 234, 463-467 (1993).

14. S. Matsuo and M. Kiuchi, "Low temperature chemical vapor deposition method utilizing an ECR plasma," Jpn. J. Appl. Phys. 22, L210-L212 (1983).

15. M. R. Alexander, R. D. Short, and F. R. Jones, "An x-ray photoelectron spectroscopic investigation into the chemical structure of deposits formed from hexamethyldisiloxane/oxygen plasmas," J. Mater. Sci. 31, 1879-1885 (1996).

16. H. A. Macleod, Thin Film Optical Filters (Macmillan, New York, 1986)

17. T. C. Paulick, "Inversion of normal-incidence (R, T) measurements to obtain $n+i k$ for thin films," Appl. Opt. 25, 562-564 (1986).

18. C. A. Mack, "Swing curves," Microlithogr. World 3(3), 23-25 (1994). 